\title{
Improvement for Amelioration Inventory Model with Weibull Distribution
}

\author{
Han-Wen Tuan, ${ }^{1}$ Shu-Cheng Lin, ${ }^{2}$ and Peterson Julian ${ }^{3}$ \\ ${ }^{1}$ Department of Computer Science and Information Management, Hungkuang University, Taichung City, Taiwan \\ ${ }^{2}$ Department of Hotel Management, Lee-Ming Institute of Technology, New Taipei City, Taiwan \\ ${ }^{3}$ Department of Traffic Science, Central Police University, Taoyuan City, Taiwan \\ Correspondence should be addressed to Peterson Julian; petersonjulian44328@gmail.com
}

Received 2 January 2017; Revised 11 May 2017; Accepted 16 May 2017; Published 12 June 2017

Academic Editor: Yakov Strelniker

Copyright @ 2017 Han-Wen Tuan et al. This is an open access article distributed under the Creative Commons Attribution License, which permits unrestricted use, distribution, and reproduction in any medium, provided the original work is properly cited.

Most inventory models dealt with deteriorated items. On the contrary, just a few papers considered inventory systems under amelioration environment. We study an amelioration inventory model with Weibull distribution. However, there are some questionable results in the amelioration paper. We will first point out those questionable results in the previous paper that did not derive the optimal solution and then provide some improvements. We will provide a rigorous analytical work for different cases dependent on the size of the shape parameter. We present a detailed numerical example for different ranges of the sharp parameter to illustrate that our solution method attains the optimal solution. We developed a new amelioration model and then provided a detailed analyzed procedure to find the optimal solution. Our findings will help researchers develop their new inventory models.

\section{Introduction}

Several published theses have addressed the importance of the deterioration phenomenon in field applications; as a result, many deterioration models have been consequently developed. Yet we have not observed much appreciation of ameliorating consideration. Due to lack of considering the influence of demand, the ameliorating items assuming duration for the amount of inventory will gradually increase; meanwhile, in the traditional inventory model dealing with deteriorating items the amount of inventory will gradually decrease. Amid the published literatures, scholars and researchers do not pay much attention to the ameliorating problems and items. To cope with this deficiency, lately a few studies are concerned with the problems of amelioration, because they do exist in the real world such as the farming, fishery, and poultry industries. The fast growing animals like ducks, pigs, and broilers in poultry farms, highbred fishes in ponds, and the cultivation of vegetables and fruits in farms are typical field applications. This is quite different from the deteriorating items and deserves a comprehensive study.

Weibull distribution has been applied to the problems of product life cycle in recent years, because it can describe the different life spans effectively by utilizing the changes of parameters. Many scholars have applied the inventory model that follows Weibull distribution. Jalan et al. [1], Chakrabarty et al. [2], and $\mathrm{Wu}$ et al. [3] considered the deteriorating inventory model. Chang and Dye [4], Wu [5], and Giri et al. [6] extended the inventory model with deteriorating items in several directions. Hwang $[7,8]$ and Mondal et al. [9] developed the inventory models with ameliorating items under various situations. Since World War II, inventory systems with the deterioration satisfying Weibull distribution have been successfully modeled in field operations; therefore, many theses are published to address the importance. Each of these extended models is applicable to certain cases in real operating applications. Some are more generally suitable than others, but most of them carry the weakness of being too complicated to control, both algebraically and numerically. Consequently, some modelers must trade off precision with respect to tractability. Hwang [7] is one of first authors to consider the remodeling from deterioration to amelioration. However, his published literatures did not present the ultimately optimal solution. Instead, he used a graphical procedure of Gupta [10] to estimate the optimal cycle time, which could not derive to a convincingly optimal solution. 
TABLE 1: Pertinent literature on amelioration inventory models with various setting.

\begin{tabular}{|c|c|c|c|c|c|c|}
\hline Authors & $\begin{array}{l}\text { Inventory } \\
\text { model }\end{array}$ & $\begin{array}{l}\text { Objective } \\
\text { function }\end{array}$ & Solution & Type & Distribution & Restriction \\
\hline Hwang [7] & EOQ & Approximated & Graphical & Amelioration & Weibull & $C_{p}>C_{a}$ \\
\hline Mondal et al. [9] & EOQ & Exact & Exact & Amelioration/deterioration & Weibull & $\begin{array}{l}\text { Demand depends on } \\
\text { price }\end{array}$ \\
\hline Moon et al. [11] & EOQ & Exact & Exact & Amelioration/deterioration & Constant & $\begin{array}{c}\text { Time-value of } \\
\text { money }\end{array}$ \\
\hline Law and Wee [12] & EPQ & Approximated & Approximated & Amelioration/deterioration & Weibull & \\
\hline Chou et al. [13] & EOQ & Exact & Exact & Amelioration & Weibull & $C_{p}>C_{a}$ \\
\hline Ji [14] & EOQ & Exact & Numerical & Amelioration/deterioration & Constant & $\begin{array}{l}\text { Time-value of } \\
\text { money }\end{array}$ \\
\hline $\begin{array}{l}\text { Valliathal and } \\
\text { Uthayakumar [15] }\end{array}$ & EPQ & Exact & Numerical & Amelioration/deterioration & Linear & $\begin{array}{l}\text { Time-value of } \\
\text { money }\end{array}$ \\
\hline Chen [16] & EOQ & Exact & Exact & Amelioration/deterioration & Constant & \\
\hline $\begin{array}{l}\text { De Zoysa and } \\
\text { Rupasinghe [17] }\end{array}$ & EOQ & Exact & Numerical & Amelioration & Linear & Linear demand \\
\hline Nodoust et al. [18] & EPQ & Exact & Approximated & Amelioration/deterioration & Constant & Linear demand \\
\hline Our manuscript & EOQ & Exact & Exact & Amelioration & Weibull & $C_{a}>C_{p}$ \\
\hline
\end{tabular}

We hereby generate a complete analytical framework to develop the optimal solution, in which both precision and tractability will be secure.

For the recent development of amelioration inventory models, we provide a list to compare 12 papers in Table 1.

\section{Notation and Assumption}

This note uses the same notation and expressions as Hwang [7] together with two auxiliary functions and several new expressions to clearly indicate our results, except that in Hwang [7], he assumed that $C_{p}>C_{a}$. However, the purchase cost of baby fry or chicken or vegetable seeds is usually less than that of feed and fertilizer, implying that $C_{p}<C_{a}$. For this note, we will discuss inventory model for items of ameliorating items with Weibull distribution, under the restriction $C_{p}<C_{a}$. For a detailed graphical expression of Weibull distribution, please refer to Wikipedia.

$R:$ the constant demand rate (kg/day)

$t$ : the parameter to denote the time of amelioration (day)

$C_{o}$ : the ordering cost (

$C_{a}$ : the amelioration cost $(W / \mathrm{kg})$

$C_{p}$ : the purchase cost $(\$ / \mathrm{kg})$, with $C_{p}<C_{a}$

$C_{h}$ : the holding cost ( $\$ / \mathrm{kg} /$ day)

$A(t)$ : the deterioration rate with $A(t)=\alpha \beta t^{\beta-1}$ (1/ day), where $\alpha$ is the shape parameter and $\beta$ is the scale parameter

$I(t)$ : the inventory level $(\mathrm{kg})$

$T$ : the cycle time (day)
TC(T): the average total cost per unit time that consists of ordering cost, purchasing cost, holding cost, and the ameliorating cost ( $\$$ day)

$f(T)$ : an auxiliary function with $d \mathrm{TC}(T) / d T=(R /$ $\left.T^{2}\right) f(T)$

$g(T)$ : another auxiliary function with $(d / d T) f(T)=$ $\alpha \beta T^{\beta} e^{-\alpha T^{\beta}} g(T)$

$T^{\#}$ : the unique positive solution for $(d / d T) g(T)=0$ with $T^{\#}=(2(1-\beta) / \alpha \beta)^{1 / \beta}$

$T^{*}$ : the optimal solution (day)

\section{Review of Previous Results}

Hwang [7] first found the initial inventory level, $I_{0}$, and then the inventory level, $I(t)$, as follows:

$$
\begin{aligned}
I_{0} & =R \int_{0}^{T} e^{-\alpha t^{\beta}} d t \\
I(t) & =e^{\alpha t^{\beta}}\left(-R \int_{0}^{t} e^{-\alpha x^{\beta}} d x+I_{0}\right) .
\end{aligned}
$$

We cite Figure 1 from Hwang [7] to illustrate the inventory level, $I(t)$.

He expressed the objection function, TC(T), as

$$
\mathrm{TC}(T)=I_{0}\left(\frac{C_{p}-C_{a}}{T}+\frac{C_{h}}{2}\right)+R C_{a}+\frac{C_{o}}{T} .
$$

Hwang used Taylor's series expansion for the exponential function to derive

$$
I_{0}=R \sum_{k=0}^{\infty} \frac{(-\alpha)^{k} T^{k \beta+1}}{k !(k \beta+1)} .
$$




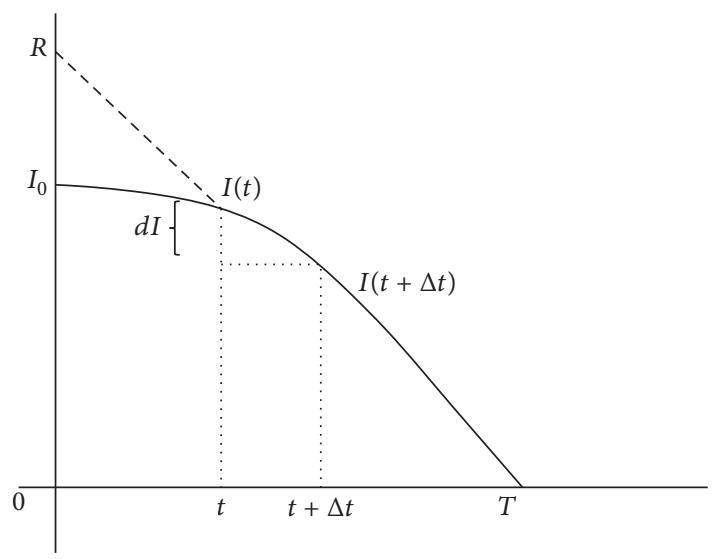

FIGURE 1: Inventory cycle of EOQ model [reproduction from Hwang [7]].

He plugged (4) into (3) and then computed $d \mathrm{TC}(T) / d T$ to imply

$$
\begin{aligned}
\frac{d \mathrm{TC}(T)}{d T}= & \left(C_{p}-C_{a}\right) R \sum_{k=0}^{\infty} \frac{(-\alpha)^{k} k \beta}{k !(k \beta+1)} T^{k \beta+1} \\
& +\frac{C_{h}}{2} R \sum_{k=0}^{\infty} \frac{(-\alpha)^{k}(k \beta+1)}{k !} T^{k \beta}-\frac{C_{o}}{T^{2}} .
\end{aligned}
$$

From (5), he used a graphical procedure of Gupta [10] as follows:

$$
T=\frac{R}{C_{o}} \sum_{k=0}^{\infty} \frac{(-k)^{k} T^{k \beta-3}}{k !}\left[\left(C_{p}-C_{a}\right) \frac{k \beta T}{k \beta+1}+\frac{C_{h}}{2}\right] .
$$

He claimed that (6) can be solved by a computer search.

\section{Discussion of Hwang's Results}

We first review his results. From the differential equation for the inventory level, we know that

$$
\frac{d}{d t} I(t)=\alpha \beta t^{\beta-1} I(t)-R
$$

where the ending inventory level is zero as $I(T)=0$; then

$$
I(t)=e^{\alpha t^{\beta}} R \int_{t}^{T} e^{-\alpha x^{\beta}} d x
$$

From the inventory level in (8), we obtain the initial inventory level as

$$
I_{0}=I(0)=R \int_{0}^{T} e^{-\alpha x^{\beta}} d x
$$

Comparing (2) and (8), we have a simple expression. For the sake of completeness, we point out that (5) should be revised as

$$
\begin{aligned}
\frac{d \mathrm{TC}(T)}{d T}= & \left(C_{p}-C_{a}\right) R \sum_{k=0}^{\infty} \frac{(-\alpha)^{k} k \beta}{k !(k \beta+1)} T^{k \beta-1} \\
& +\frac{C_{h}}{2} R \sum_{k=0}^{\infty} \frac{(-\alpha)^{k}}{k !} T^{k \beta}-\frac{C_{o}}{T^{2}}
\end{aligned}
$$

Moreover, (6) should be modified as

$$
\begin{aligned}
T= & \frac{R}{C_{o}} \sum_{k=0}^{\infty} \frac{(-\alpha)^{k} T^{k \beta+3}}{k !} \\
& \cdot\left[\left(C_{p}-C_{a}\right) \frac{k \beta}{(k \beta+1) T}+\frac{C_{h}}{2}\right] .
\end{aligned}
$$

Hwang [7] did not explain why (5) has solutions or the solution is unique. He just sketched a graph to show that there is a unique solution for (5) without further explanation. We will derive a series of lemmas and theorems to find out whether (5) has a unique solution.

\section{Our Improvement}

We plug (9) into (3) to take the first derivative; then it yields that

$$
\frac{d \mathrm{TC}(T)}{d T}=\frac{R}{T^{2}} f(T)
$$

with an auxiliary function, say $f(T)$, where

$$
\begin{aligned}
f(T)= & \left(C_{a}-C_{p}\right)\left[\int_{0}^{T} e^{-\alpha x^{\beta}} d x-T e^{-\alpha T^{\beta}}\right] \\
& +\frac{C_{h}}{2} T^{2} e^{-\alpha T^{\beta}}-\frac{C_{o}}{R} .
\end{aligned}
$$

Studying the properties of $d \mathrm{TC}(T) / d T$ is equivalent to examining the properties of $f(T)$. We obtain that

$$
\frac{d}{d T} f(T)=\alpha \beta T^{\beta} e^{-\alpha T^{\beta}} g(T)
$$

with another auxiliary function, say $g(T)$, where

$$
g(T)=\frac{C_{h}}{\alpha \beta} T^{1-\beta}-\frac{C_{h}}{2} T+\left(C_{a}-C_{p}\right) .
$$

According to the expression in (15), we divide the inventory model into the following three cases: (a) $0<\beta<1$, (b) $\beta=1$, and (c) $\beta>1$.

For the first case with $0<\beta<1$, motivated by (15), it follows that

$$
\frac{d}{d T} g(T)=\frac{C_{h}(1-\beta)}{\alpha \beta}\left(\frac{1}{T^{\beta}}-\frac{\alpha \beta}{2(1-\beta)}\right) .
$$


To simplify the expression, we assume the unique positive root of (16) as $T^{\#}$, which means

$$
T^{\#}=\left(\frac{2(1-\beta)}{\alpha \beta}\right)^{1 / \beta}
$$

From (16), we know that $(d / d T) g(T)>0$ for $0<T<T^{\#}$ and $(d / d T) g(T)<0$ for $T>T^{\#}$. Hence, $g(T)$ increases for $0<$ $T<T^{\#}$ and $g(T)$ decreases for $T>T^{\#}$.

From $g(0)=C_{a}-C_{p}>0, g(T)$ increases to $g\left(T^{\#}\right)$ then $g(T)$ decreases to $g(\infty)=-\infty$, such that there is a unique point, say $T_{1}$, with $g\left(T_{1}\right)=0$ as

$$
\frac{C_{h}}{\alpha \beta} T_{1}^{1-\beta}+\left(C_{a}-C_{p}\right)=\frac{C_{h}}{2} T_{1} .
$$

It yields that $g(T)>0$ for $0<T<T_{1}$ and $g(T)<0$ for $T_{1}<T$. From (13), we obtain that $f(0)=-C_{0} / R<0$. By (14), it implies that $f(T)$ increases for $0<T<T_{1}$ and $f(T)$ decreases for $T_{1}<T$.

Using (18), we obtain that

$$
\begin{aligned}
f\left(T_{1}\right)= & \left(C_{a}-C_{p}\right) \int_{0}^{T_{1}} e^{-\alpha x^{\beta}} d x+\frac{C_{h}}{\alpha \beta} T_{1}{ }^{2-\beta} e^{-\alpha T_{1}{ }^{\beta}} \\
& -\frac{C_{o}}{R} .
\end{aligned}
$$

Depending on the value of $f\left(T_{1}\right)$ and $f(\infty)$, we will divide case (a) into three cases: (a1) $f\left(T_{1}\right) \leq 0$, (a2) $f\left(T_{1}\right)>0$ and $f(\infty)<0$, and (a3) $f\left(T_{1}\right)>0$ and $f(\infty) \geq 0$.

Lemma 1. For case (a1), $f\left(T_{1}\right) \leq 0$ and then the optimal solution $T^{*}=\infty$.

From $f(T) \leq 0$ for $T>0$, by (12), $d T C(T) / d T \leq 0$ for $T>0$ so the minimum value will occur when $T \rightarrow \infty$.

Next, we consider case (a2). Since $f\left(T_{1}\right)>0$, then $f(T)$ increases from $f(0)=-C_{0} / R<0$ to $f\left(T_{1}\right)>0$ for $0<T<T_{1}$ and $f(T)$ decreases from $f\left(T_{1}\right)>0$ to $f(\infty)<0$. Hence, there are two points, say $T_{2}$ and $T_{3}$, where $0<T_{2}<T_{1}<T_{3}$ with $f\left(T_{2}\right)=0$ and $f\left(T_{3}\right)=0$. It yields that $f(T)<0$, for $0<$ $T<T_{2}, f(T)>0$ for $T_{2}<T<T_{3}$, and $f(T)<0$, for $T_{3}<T$. Consequently, by (12), TC(T) decreases for $0<T<T_{2}$, then $\mathrm{TC}(T)$ increases for $T_{2}<T<T_{3}$, and then TC $(T)$ decreases for $T_{3}<T$. Hence, we derive that $T=0$ and $T=T_{3}$ are local maximum points. On the other hand, $T=T_{2}$ and $T=\infty$ are local minimum points. We summarize our finding in the next lemma.

Lemma 2. For case (a2), the minimum value of $T C(T)$ is $\min \left\{T C\left(T_{2}\right), T C(\infty)\right\}$.

Third, we consider case (a3). From $f\left(T_{1}\right)>0$ and $f(\infty) \geq$ 0 , we know that there exists a unique point, say $T_{4}$ such that $f\left(T_{4}\right)=0$ satisfying $0<T_{4}<T_{1}$. It follows that $f(T)<0$, for $0<T<T_{4}$ and $f(T)>0$ for $T_{4}<T$. By the same argument, TC $(T)$ decreases for $0<T<T_{4}$ and then TC $(T)$ increases for $T_{4}<T$ such that $T_{4}$ is the unique local minimum point.
Lemma 3. For case (a3), the minimum value of $T C(T)$ is $\mathrm{TC}\left(\mathrm{T}_{4}\right)$.

We combine all previous results in the next main theorem.

Theorem 4. Under the condition $0<\beta<1$, for the minimum point, $T^{*}$, and minimum value, $T C\left(T^{*}\right)$, there are three different cases:

(1) For case (a1), with $f\left(T_{1}\right) \leq 0$, then the optimal solution $T^{*}=\infty$.

(2) For case (a2), with $f\left(T_{1}\right)>0$ and $f(\infty)<0$, the minimum value of TC(T) is $\min \left\{T C\left(T_{2}\right), T C(\infty)\right\}$.

(3) For case (a3), with $f\left(T_{1}\right)>0$ and $f(\infty) \geq 0$, the minimum value of TC(T) is TC $\left(T_{4}\right)$.

To help researchers absorb our findings, we sketch four different cases from Theorem 4 in Figure 2.

Now, we begin to discuss the second case with $\beta=1$. From (15), we know that

$$
g(T)=\frac{C_{h}}{\alpha}+\left(C_{a}-C_{p}\right)-\frac{C_{h}}{2} T .
$$

To simplify the expression, we assume that

$$
T_{5}=\frac{2}{\alpha}+\frac{2\left(C_{a}-C_{p}\right)}{C_{h}} .
$$

We know that $g(T)>0$ for $T<T_{5}$ and $g(T)<0$ for $T_{5}<T$. It implies that $f(T)$ increases for $T<T_{5}$ and $f(T)$ decreases for $T_{5}<T$.

Depending on the value of $f\left(T_{5}\right)$ and $f(\infty)$, we will divide case (b) into three cases: (b1) $f\left(T_{5}\right) \leq 0$, (b2) $f\left(T_{5}\right)>0$ and $f(\infty)<0$, and (b3) $f\left(T_{5}\right)>0$ and $f(\infty) \geq 0$.

By the same argument for the case of $0<\beta<1$ to replace $T_{1}$ by $T_{5}$, we may derive the similar theorem as follows, where $T_{6}$ and $T_{7}$ are the roots for $f(T)=0$ under case (b2) where $0<T_{6}<T_{5}<T_{7}$ and $T_{8}$ is the root for $f(T)=0$ under case (b3).

Theorem 5. Under the condition $\beta=1$, for the minimum point, $T^{*}$, and minimum value, $T C\left(T^{*}\right)$, there are three different cases:

(1) For case (b1) with $f\left(T_{5}\right) \leq 0$, then the optimal solution $T^{*}=\infty$.

(2) For case (b2), with $f\left(T_{5}\right)>0$ and $f(\infty)<0$, the minimum value of TC $(T)$ is $\min \left\{T C\left(T_{6}\right), T C(\infty)\right\}$.

(3) For case (b3), with $f\left(T_{5}\right)>0$ and $f(\infty) \geq 0$, the minimum value of TC $(T)$ is $T C\left(T_{8}\right)$.

To help researchers absorb our findings, we sketch four different cases from Theorem 5 in Figure 3.

At last, we begin to discuss the third case with $\beta>1$. From (15), we know that

$$
\frac{d}{d T} g(T)=\frac{-C_{h}(\beta-1)}{\alpha \beta}\left(\frac{1}{T^{\beta}}+\frac{\alpha \beta}{2(\beta-1)}\right)<0,
$$




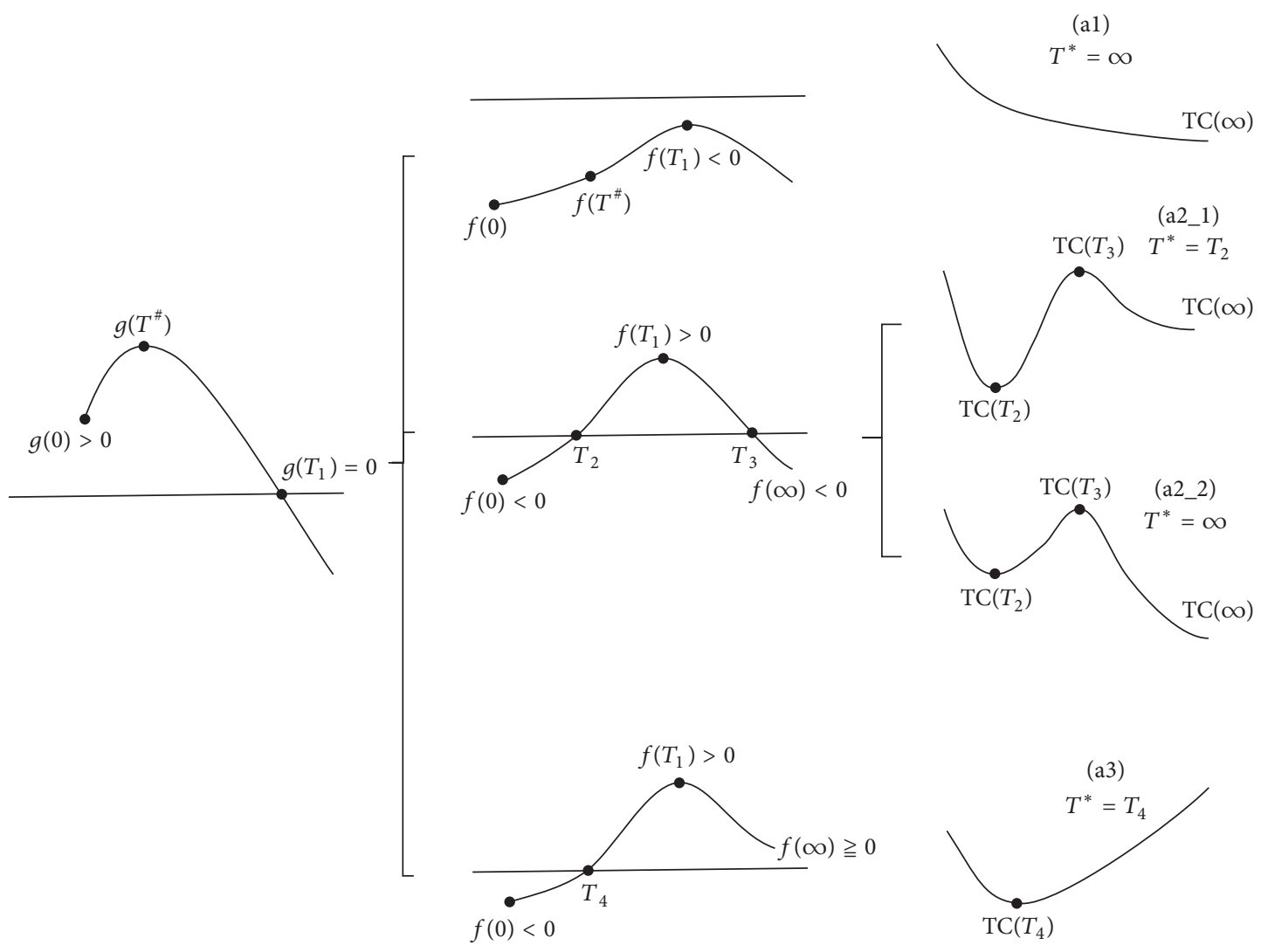

FIGURE 2: For $0<\beta<1$, four cases are illustrated. The sign of $f\left(T_{1}\right)$ is first considered for branching, and the sign of $f(\infty)$ is then considered for further branching. The value of $\mathrm{TC}\left(T^{*}\right)$ is lastly considered to result in cases (a2_1) and (a2_2).

so $g(T)$ is a decreasing function from $\lim _{T \rightarrow 0^{+}} g(T)=\infty$ to $\lim _{T \rightarrow \infty} g(T)=-\infty$; then there is a unique point, say $T^{\$}$, with $g\left(T^{\$}\right)=0$ and $g(T)>0$ for $0<T<T^{\$}$ and $g(T)<0$ for $T>T^{\$}$. It implies that $f(T)$ increases for $0<T<T^{\$}$ and $f(T)$ decreases for $T>T^{\$}$.

Depending on the value of $f\left(T^{\$}\right)$ and $f(\infty)$, we will divide case (c) into three cases: (c1) $f\left(T^{\$}\right) \leq 0,(\mathrm{c} 2) f\left(T^{\$}\right)>0$ and $f(\infty)<0$, and $(\mathrm{c} 3) f\left(T^{\$}\right)>0$ and $f(\infty) \geq 0$.

By the same argument for the case of $0<\beta<1$ to replace $T_{1}$ by $T^{\$}$, we may derive similar theorem as follows, where $T_{9}$ and $T_{10}$ are the roots for $f(T)=0$ under case (c2) where $0<T_{9}<T^{\$}<T_{10}$ and $T_{11}$ is the root for $f(T)=0$ under case (c3).

Theorem 6. Under the condition $\beta>1$, for the minimum point, $T^{*}$, and minimum value, $T C\left(T^{*}\right)$, there are three different cases:

(1) For case (c1) with $f\left(T^{\$}\right) \leq 0$, then the optimal solution $T^{*}=\infty$.

(2) For case (c2), with $f\left(T^{\$}\right)>0$ and $f(\infty)<0$, the minimum value of $T C(T)$ is $\min \left\{T C\left(T_{9}\right), T C(\infty)\right\}$.

(3) For case (c3), with $f\left(T^{\$}\right)>0$ and $f(\infty) \geq 0$, the minimum value of $T C(T)$ is $T C\left(T_{11}\right)$.
To help researchers absorb our findings, we sketch four different cases from Theorem 6 in Figure 4 .

Motivated by Figures 2-4, for $0<\beta<1, \beta=1$, and $\beta>1$, respectively, we know that graphs of $g(T)$ are different for three different cases. However, we can abstractly combine these three different graphs of $g(T)$ to imply that there is a unique point, say $T^{\#}$, such that $g(T)>0$, for $0<T<T^{\#}$, and $g(T)<0$, for $T>T^{\#}$. Consequently, we observe that cases (a1), (b1), and (cl) are of the same type. Similarly, cases (a2), (b2), and (c2) looked like each other. Moreover, cases (a3), (b3), and (c3) are similar. Hence, we claim that there is a unique point, say $T^{\#}$, satisfying $g\left(T^{\#}\right)=0$ and there is a unique point, say $T^{\Delta}$, with $0<T^{\Delta}<T^{\#}$ and $f\left(T^{\Delta}\right)=0$; we can merge Theorems $4-6$ into a general result as follows.

Theorem 7. For our amelioration model with $C_{a}>C_{p}$, we obtain that first solve $g(T)=0$ to yield the unique solution $T^{\#}$. Checking the value of $f\left(T^{\#}\right)$, we divide the solution procedure into two different cases.

(a) If $f\left(T^{\#}\right) \leq 0$, then the optimal solution $T^{*}=\infty$.

(b) If $f\left(T^{\#}\right)>0$, then there is a unique point, say $T^{\Delta}$, with $0<T^{\Delta}<T^{\#}$ and $f\left(T^{\Delta}\right)=0$; then the minimum value is $\min \left\{T C\left(T^{\Delta}\right), T C(\infty)\right\}$. 


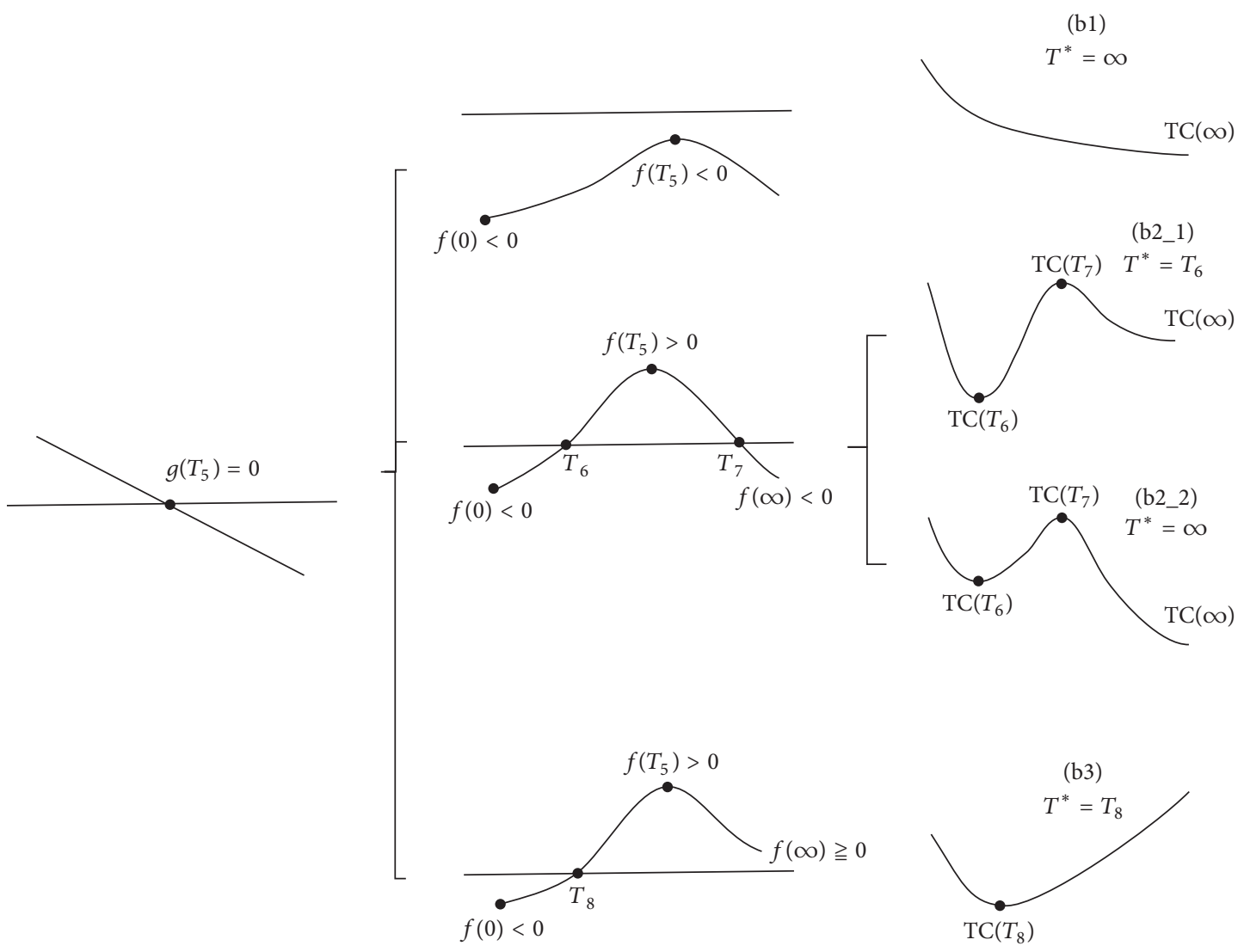

FIGURE 3: For $\beta=1$, four cases are illustrated. The sign of $f\left(T_{5}\right)$ is first considered for branching, and the sign of $f(\infty)$ is then considered for further branching. The value of $\operatorname{TC}\left(T^{*}\right)$ is lastly considered to result in cases (b2_1) and (b2_2).

\section{Numerical Examples}

To be comparable with the results in Hwang [7], we consider the same numerical example as Example 1 in it with the following data: $R=1000, C_{h}=400, C_{o}=300000, C_{p}=10000$, and $\alpha=0.05$, except that we change $C_{a}$ from 4000 to 40000 such that we have $C_{a}>C_{p}$ and $\beta$ from 0.05 into three different cases: in Example 1, $\beta=0.05$, in Example $2, \beta=1$, and in Example 3, $\beta=2$ such that it provides three examples for each case, respectively.

Example 1. Under the condition $\beta=0.05$, that is suitable for case (a), we have that $T_{1}=1.153 \times 10^{58}, f\left(T_{1}\right)>0$, and $f(\infty)>0$; by case (a3), the optimal solution is $T_{4}=$ 1.0889806138 and the optimal value is $\operatorname{TC}\left(T_{4}\right)=1.1883967 \times$ $10^{7}$.

Example 2. Under the condition $\beta=1$, that is suitable for case (b), we obtain that $T_{5}=190, f\left(T_{5}\right)>0$ and $f(\infty)>0$; from case (b3), the optimal solution is $T_{8}=0.56785328$ and the optimal value is $\operatorname{TC}\left(T_{8}\right)=1.1062167 \times 10^{7}$.

Example 3. Under the condition $\beta=2$, that is suitable for case (c), we find that $T^{\$}=150.133, f\left(T^{\$}\right)>0$, and $f(\infty)>0$; from case (c3), the optimal solution is $T_{11}=0.611864431$ and the optimal value is $\mathrm{TC}\left(T_{11}\right)=1.0798061 \times 10^{7}$.
Under our classification to different cases, we derive the optimal cycle time and the optimal total cost that will help the researcher and practitioners to obtain the best decision making.

For practitioners easily applying our results, we provide a detailed algorithm for the optimal solution and the optimal value.

Step 1. Solve $g(T)=0$ to derive the unique root, say $T^{\#}$.

Step 2. Compute $f\left(T^{\#}\right)$.

Step 3. If $f\left(T^{\#}\right) \leq 0$, then the optimal solution is $T^{*}=\infty$.

Step 4. If $f\left(T^{\#}\right)>0$, then solve $f(T)=0$ to locate the unique root, say $T^{\Delta}$, satisfying $0<T^{\Delta}<T^{\#}$; then the optimal value $=\min \left\{\mathrm{TC}\left(T^{\Delta}\right), \mathrm{TC}(\infty)\right\}$.

In the following, we provided a comparison between two restrictions: (a) In Hwang [7] and Chou et al. [13] with $C_{p}>$ $C_{a}$ and (b) in our manuscript with $C_{a}>C_{p}$. For eel, until now, no one can produce baby eel from eggs of a pregnant female eel. Hence, the baby eel must be caught from the wild and then sold by tail, for example, $\$ 2 /$ per tail, and then the purchasing cost (per kilogram) $C_{p}$ will be very large. The selling price for an adult eel, for example, is $\$ 10 /$ per tail such that $C_{p}>C_{a}$ 

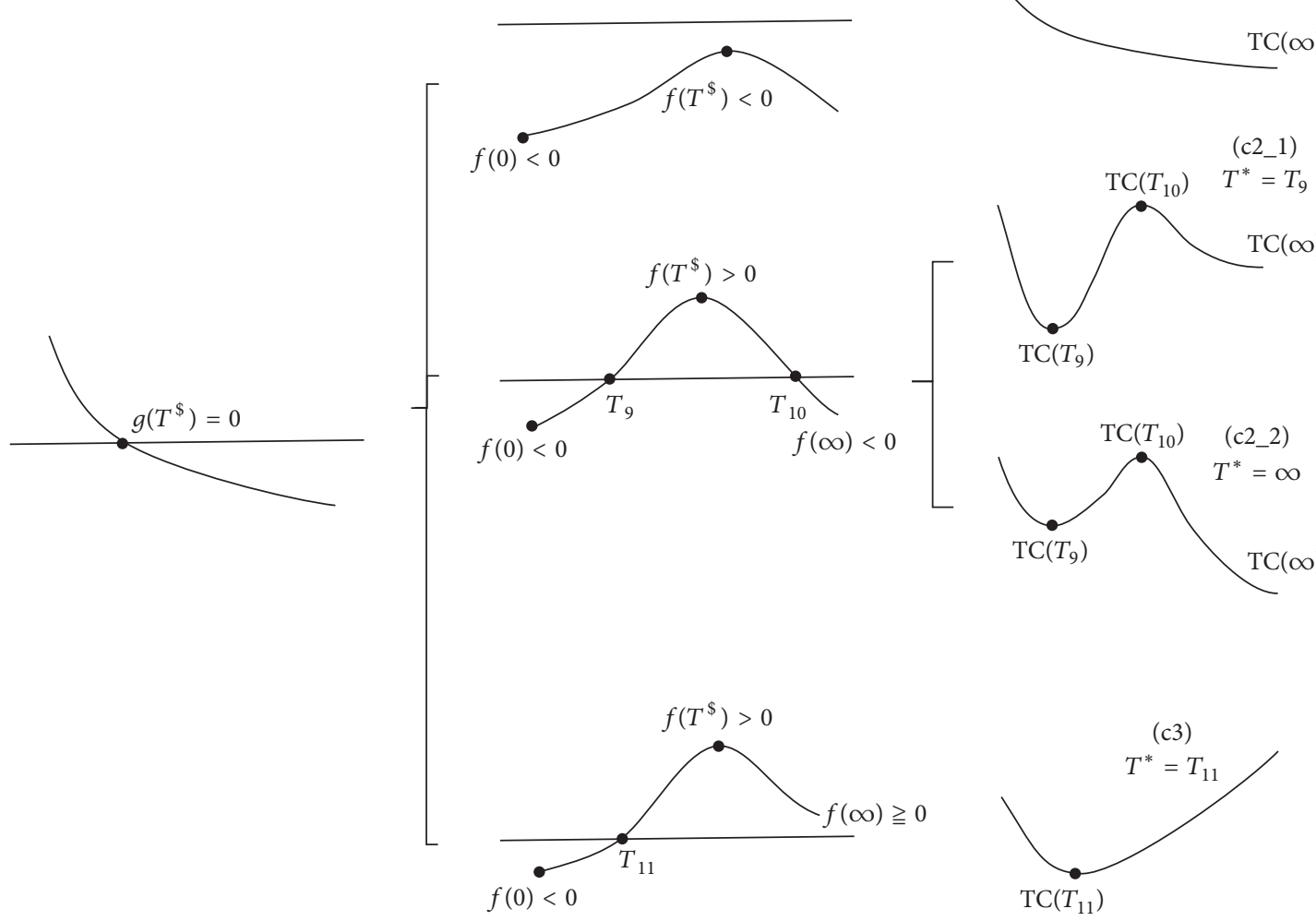

FIgURE 4: For $\beta>1$, four cases are illustrated. The sign of $f\left(T^{\$}\right)$ is first considered for branching, and the sign of $f(\infty)$ is then considered for further branching. The value of $\mathrm{TC}\left(T^{*}\right)$ is lastly considered to result in cases (c2_1) and (c2_2).

(in kilogram) is valid for eel that is consistent with Hwang [7] and Chou et al. [13].

On the other hand, from Wikipedia, there is a picture that shows "Expressing eggs from a female rainbow trout" indicating that rainbow trout can be reproduced from eggs such that the purchasing cost for rainbow trout will be low and then our assumption $C_{a}>C_{p}$ can be applied for this kind of fish farming.

Hence, amelioration inventory models can be used to measure (a) weight gain or (b) pregnant mother fish delivering new born baby fish.

\section{Direction for the Future Research}

There are two amelioration inventory models in Hwang [7]. For the first model, the demand rate is greater than the amelioration rate. For the second model, the amelioration rate is greater than the demand rate. Under this restriction, Hwang [7] developed his "partially selling quantity model" which can be treated as sustainable management. Hence, we can predict that researchers may consider the exact objective function and then try to derive the optimal solution that will be an interesting research topic in the future.

\section{Conclusion}

With careful classification based on theoretical constructions, this paper developed an analytical framework for Weibull ameliorating in inventory models and provided the optimal solution of cycle time and the minimum value of average total cost. Numerical examples were also provided to illustrate our findings.

\section{Conflicts of Interest}

The authors declare that there are no conflicts of interest regarding the publication of this paper.

\section{Acknowledgments}

The authors greatly appreciate the partial financial support of MOST 105-2410-H-015-006. They express their sincere gratitude to Scarlet Fang (x666s42@gmail.com) for his help of sketching four figures.

\section{References}

[1] A. K. Jalan, R. R. Giri, and K. S. Chaudhuri, "EOQ model for items with Weibull distribution deterioration, shortages and 
trended demand," International Journal of Systems Science, vol. 27, no. 9, pp. 851-855, 1996.

[2] T. Chakrabarty, B. C. Giri, and K. S. Chaudhuri, "An EOQ model for items with weibull distribution deterioration, shortages and trended demand: an extension of philip's model," Computers and Operations Research, vol. 25, no. 7-8, pp. 649-657, 1998.

[3] J.-W. Wu, C. Lin, B. Tan, and W.-C. Lee, "An EOQ inventory model with time-varying demand and Weibull deterioration with shortages," International Journal of Systems Science, vol. 31, no. 6, pp. 677-683, 2000.

[4] H.-J. Chang and C.-Y. Dye, "An EOQ model with deteriorating items in response to a temporary sale price," Production Planning and Control, vol. 11, no. 5, pp. 464-473, 2000.

[5] K.-S. Wu, "Deterministic inventory model for items with time varying demand, Weibull distribution deterioration and shortages," Yugoslav Journal of Operations Research, vol. 12, no. 1, pp. 61-71, 2002.

[6] B. C. Giri, A. K. Jalan, and K. S. Chaudhuri, "Economic order quantity model with Weibull deterioration distribution, shortage and ramp-type demand," International Journal of Systems Science, vol. 34, no. 4, pp. 237-243, 2003.

[7] H.-S. Hwang, "A study on an inventory model for items with Weibull ameliorating," Computers and Industrial Engineering, vol. 33, no. 3-4, pp. 701-704, 1997.

[8] H.-S. Hwang, "Inventory models for both deteriorating and ameliorating items," Computers and Industrial Engineering, vol. 37, no. 1, pp. 257-260, 1999.

[9] B. Mondal, A. K. Bhunia, and M. Maiti, "An inventory system of ameliorating items for price dependent demand rate," Computers and Industrial Engineering, vol. 45, no. 3, pp. 443-456, 2003.

[10] N. K. Gupta, "Effect of lead time on inventory-A working result," Journal of the Operational Research Society, vol. 30, no. 5, pp. 477-481, 1979.

[11] I. Moon, B. C. Giri, and B. Ko, "Economic order quantity models for ameliorating/deteriorating items under inflation and time discounting," European Journal of Operational Research, vol. 162, no. 3, pp. 773-785, 2005.

[12] S.-T. Law and H.-M. Wee, "An integrated production-inventory model for ameliorating and deteriorating items taking account of time discounting," Mathematical and Computer Modelling, vol. 43, no. 5-6, pp. 673-685, 2006.

[13] S.-Y. Chou, W. T. Chouhuang, J. S. Lin, and P. Chu, "An analytical solution approach for the economic order quantity model with Weibull ameliorating items," Mathematical and Computer Modelling, vol. 48, no. 11-12, pp. 1868-1874, 2008.

[14] L.-Q. Ji, “The influences of inflation and time-value of money on an EOQ model for both ameliorating and deteriorating items with partial backlogging," in Proceedings of the 2008 International Conference on Wireless Communications, Networking and Mobile Computing, WiCOM 2008, Dalian, China, 2008.

[15] M. Valliathal and R. Uthayakumar, "The production-inventory problem for ameliorating/deteriorating items with non-linear shortage cost under inflation and time discounting," Applied Mathematical Sciences, vol. 4, no. 5-8, pp. 289-304, 2010.

[16] J. Chen, "An inventory model for ameliorating and deteriorating fresh agricultural items with ripeness and price dependent demand," in Proceedings of the 2011 International Conference on Information Technology, Computer Engineering and Management Sciences, ICM 2011, pp. 228-231, Jiangsu, China, 2011.

[17] D. C. De Zoysa and T. D. Rupasinghe, "Development of a safety stock optimization model with high demand uncertainties for
Winery Supply Chains (WSCs)," in Proceedings of the 2016 Manufacturing \& Industrial Engineering Symposium (MIES), pp. 1-5, Colombo, Sri Lanka, 2016.

[18] S. Nodoust, A. Mirzazadeh, and G. Weber, "An evidential reasoning approach for production modeling with deteriorating and ameliorating items," Operational Research, 2017. 


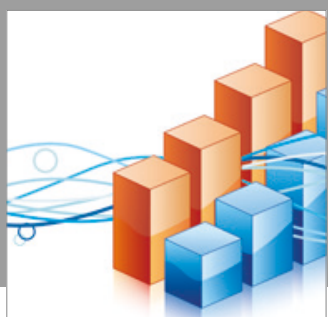

Advances in

Operations Research

vatersals

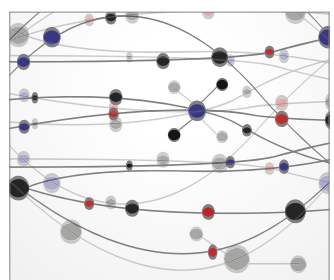

\section{The Scientific} World Journal
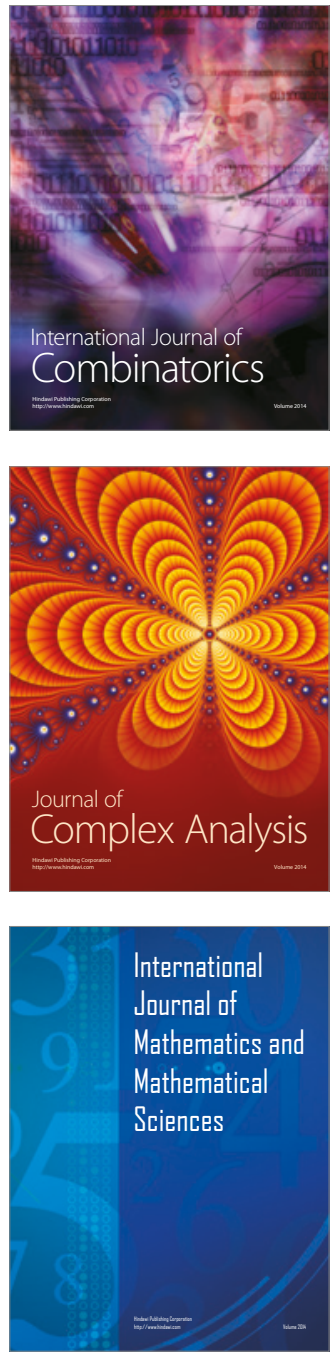
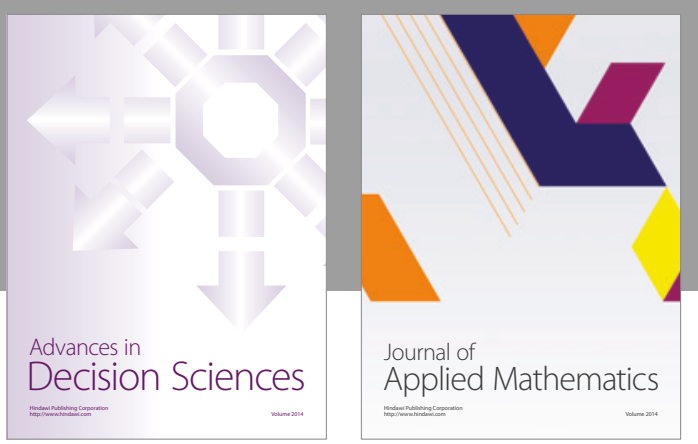

Algebra

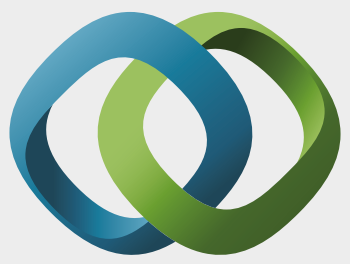

\section{Hindawi}

Submit your manuscripts at

https://www.hindawi.com
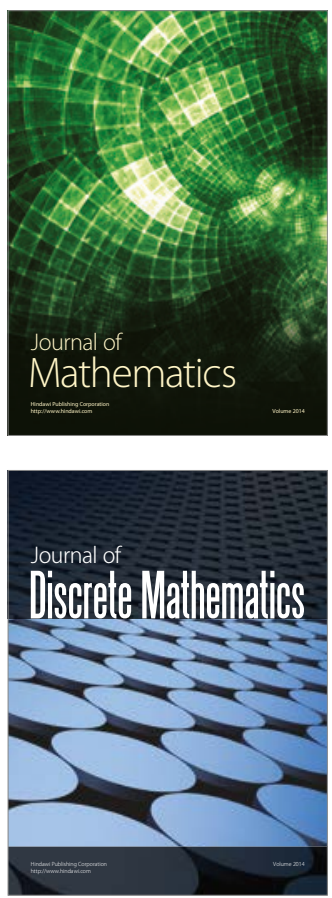

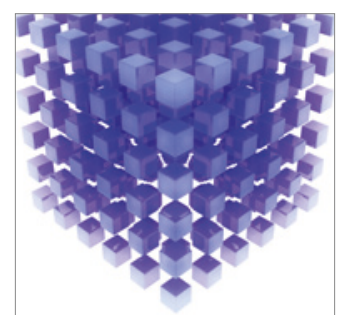

Mathematical Problems in Engineering
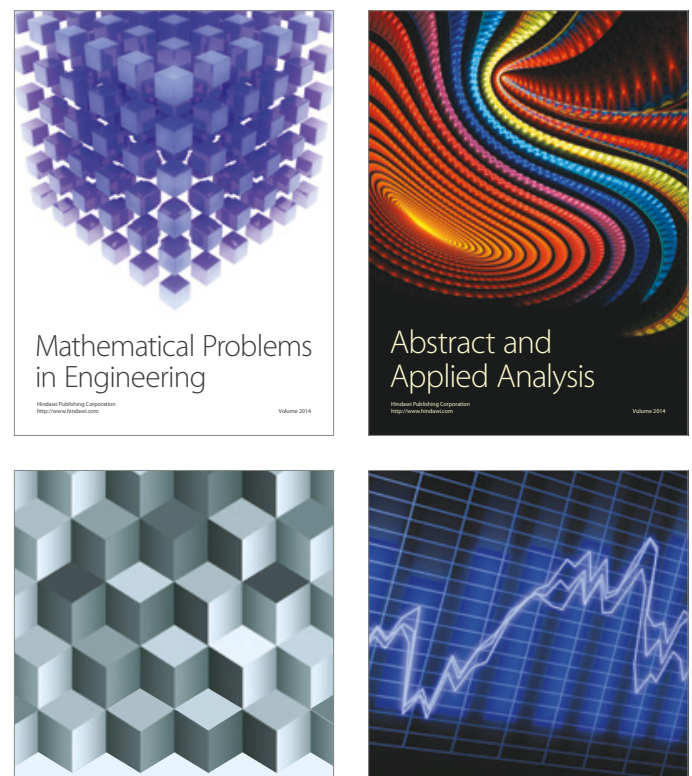

Journal of

Function Spaces

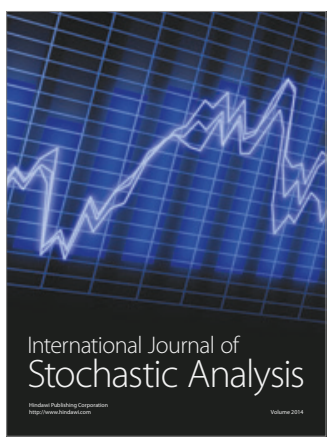

Probability and Statistics
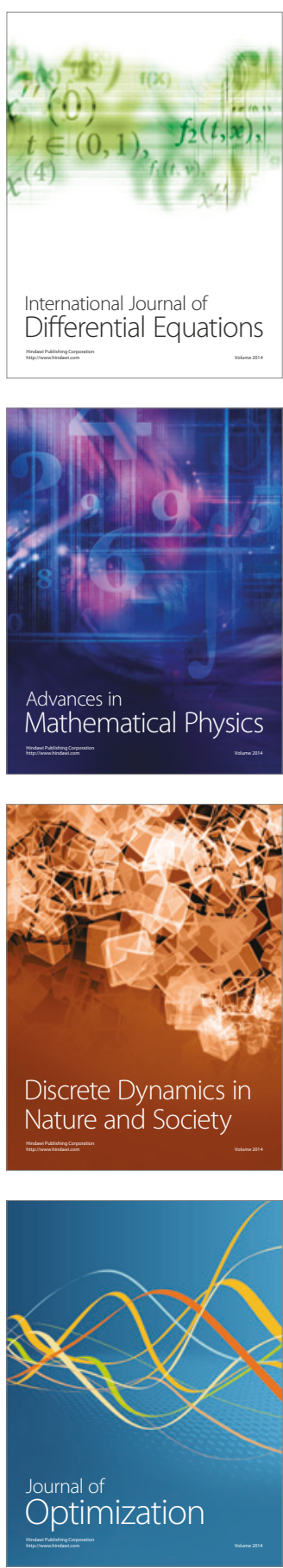